\title{
Characterisation Techniques for the Identification of Composite Cladding Materials
}

\author{
Laurel George $^{1 *}$ and Richard Wuhrer ${ }^{1}$ \\ 1. Advanced Material Characterisation Facility (AMCF), Western Sydney University, Sydney, Australia. \\ * Corresponding author: L.George@westernsydney.edu.au
}

This paper demonstrates how characterisation techniques can be utilised in solving real-world industry based problems, such as verification of building products. Recent tragedies, such as the 2017 Grenfell Tower fire in London and the 2014 Lacrosse building fire in Australia, saw the rapid spread of fire vertically up the buildings' external walls where Aluminium Composite Panels (ACPs) had been used. Since this time, the NSW Commissioner for Fair Trading in Australia examined the use of ACPs, which usually consist of a highly flammable polyethylene (PE) core, in the building industry. In August 2018 a ban was imposed on the use of ACPs with a core comprising of greater than $30 \%$ PE by mass, on all buildings greater than 3 storeys high [1]. This has led to the testing of all buildings of at least this height.

Another concern outlined in a 2018 report commissioned by the Building Minister's Forum, was the high occurrence of inferior building products [2]. It was noted that there had been incidences of product substitution in ACPs, and as it was recommended a compulsory building product certification system be put in place in the industry, particularly for high risk products. This is where characterisation techniques are invaluable in verifying that the product specified is that which has been used on a building is important, even if a product specification sheet is provided.

ACPs consist of a core material which is sandwiched between two thin aluminium sheets. The core material content falls into one of 3 categories: 1) close to $100 \% \mathrm{PE}, 2$ ) a mixture of PE and mineral fillers, and 3) less than 3\% PE [1]. Identification of the type of polymer present and mineral filler (if any) is vital, as they all act differently when exposed to heat. For example, the mineral filler aluminium hydroxide when heated above $180^{\circ} \mathrm{C}$ will decompose and release water, delaying ignition of the core.

In order to verify the core materials present and the percent fillers used, a combination of complimentary characterisation techniques can be utilised. These include; 1) Scanning Electron Microscopy (SEM) equipped with Energy Dispersive Spectroscopy (EDS) for Microanalysis, 2) Fourier Transform InfraRed (FTIR) spectroscopy, 3) X-Ray Diffraction (XRD) and 4) Thermal analysis techniques such as Thermal Gravimetric Analysis (TGA) and Differential Scanning Calorimetry (DSC).

An SEM backscatter image can first be easily used to verify if there are any fillers present in ACP core samples (Figures 1a). This image can also reveal area fraction of fillers. EDS analysis can be used to confirm what elements are present if there are any fillers in the sample (Figures 1a inset). A FTIR spectrum can then be obtained (Figure 1b), and a subsequent library search undertaken to find matches for the polymer and any fillers. Elements found in the EDS results can be used here to help narrow down search results. Materials with a mix of different components (e.g. a polymer with filler materials), can be quite hard to match, as the final spectrum obtained is a composite of the different material spectra added together. In this case, multiple library matches may need to be done in order to account for all peaks. XRD (Figure 1c) can also help the verify filler, and what type/phase in particular is present.

In order to determine the percentage mass of PE and filler material, which is needed as outlined in the 
ACP ban [1], thermal analysis such as TGA/DSC (Figures 1d and e) can be used (if required). If only one filler is present in a polymer, TGA can be used to monitor the mass of a sample as the polymer burns off, leaving a residual mass representing the mineral filler. DSC can confirm thermal events, such as melting and decomposition temperatures. Thermal techniques are not always straight forward however, and depend on how many materials are present, what temperature thermal events occur and if they overlap.

Quite often only the first technique of microscopy and microanalysis is required. However, the combination of microscopy, microanalysis and other related techniques like FTIR, XRD and Thermal Analysis can reveal further information about the materials being used [3].

References:

[1] Aluminium Composite Panel Ban, www.fairtrading.nsw.gov.au/buying-products-andservices/product -and-service-safety/building-products/aluminium-composite-panel-ban (accessed Feb 2, 2019).

[2] P Shergold and B Weir, Building Confidence, Dept. of Industry, Innovation and Science (2018).

[3] The authors thank AMCF interns Denise Duff, Daniel Fanna, Edward Huang, Kash Bhullar and Chris Rhodes.
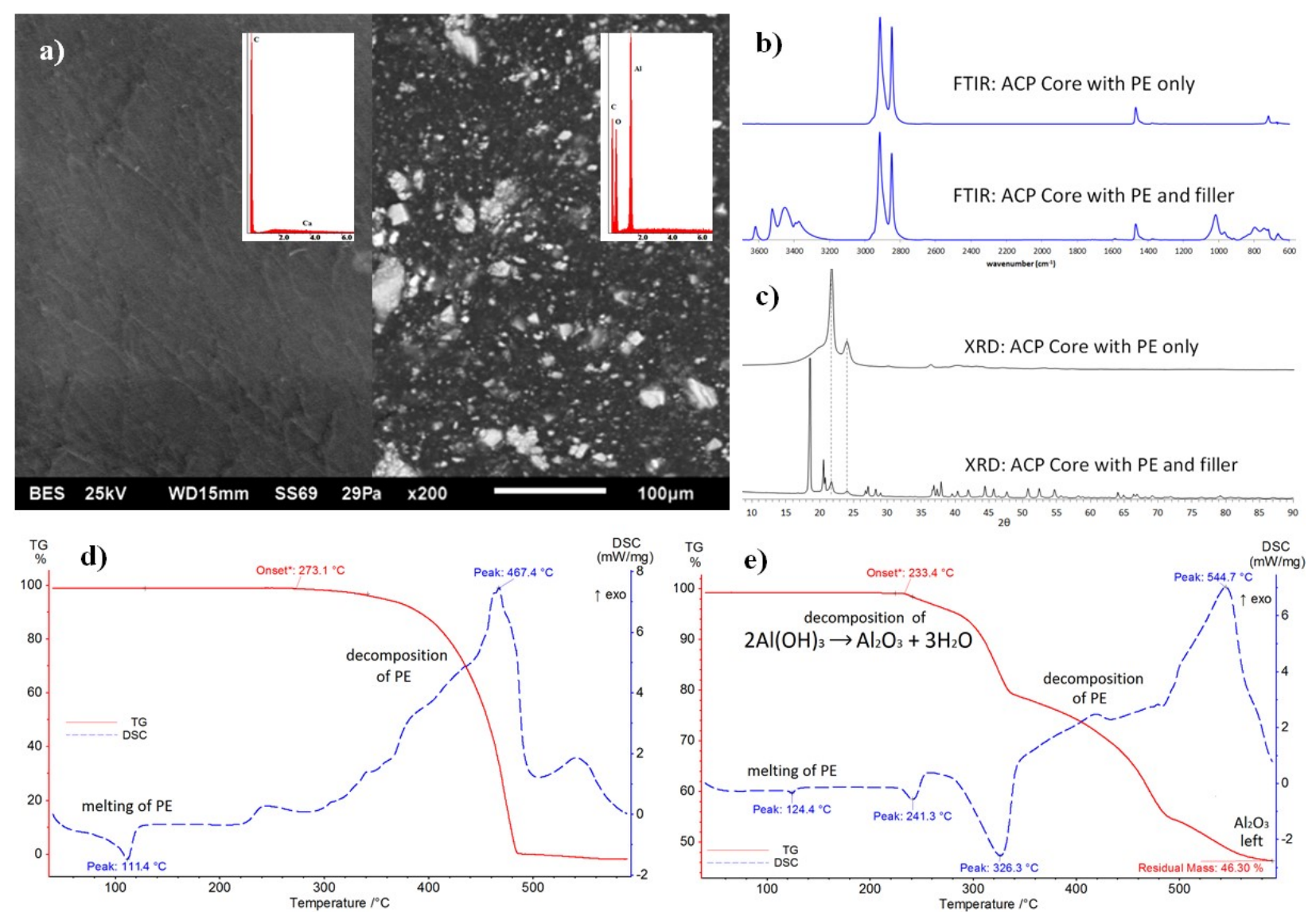

Figure 1. a) SEM BSE image with EDS insert showing elements present for a sample with only PE present (left) and a sample showing PE with fillers (right), b) FTIR spectra. c) XRD spectra, d) TGA/DSC of a core panel with only PE present and e) TGA/DSC of core panel with both PE and fillers. 